\title{
ARTICLE
}

Received 30 Jun 2016 | Accepted 4 Oct 2016 | Published 16 Nov 2016

\section{Mechanical gating of a mechanochemical reaction cascade}

\author{
Junpeng Wang ${ }^{1}$, Tatiana B. Kouznetsova ${ }^{1}$, Roman Boulatov ${ }^{2} \&$ Stephen L. Craig $^{1}$
}

Covalent polymer mechanochemistry offers promising opportunities for the control and engineering of reactivity. To date, covalent mechanochemistry has largely been limited to individual reactions, but it also presents potential for intricate reaction systems and feedback loops. Here we report a molecular architecture, in which a cyclobutane mechanophore functions as a gate to regulate the activation of a second mechanophore, dichlorocyclopropane, resulting in a mechanochemical cascade reaction. Single-molecule force spectroscopy, pulsed ultrasonication experiments and DFT-level calculations support gating and indicate that extra force of $>0.5 \mathrm{nN}$ needs to be applied to a polymer of gated $g \mathrm{DCC}$ than of free $g D C C$ for the mechanochemical isomerization $g D C C$ to proceed at equal rate. The gating concept provides a mechanism by which to regulate stress-responsive behaviours, such as load-strengthening and mechanochromism, in future materials designs.

\footnotetext{
${ }^{1}$ Department of Chemistry, Duke University, Durham, North Carolina 27708, USA. ${ }^{2}$ Department of Chemistry, University of Liverpool, Crown Street, Liverpoo L69 7ZD, UK. Correspondence and requests for materials should be addressed to R.B. (email: r.boulatov@liv.ac.uk) or to S.L.C. (email: stephen.craig@duke.edu).
} 
C ovalent polymer mechanochemistry ${ }^{1-5}$ has been extensively explored in recent years for a variety of purposes, including biasing reaction pathways ${ }^{6-9}$, trapping transition states and intermediates ${ }^{10,11}$, catalysis ${ }^{12-15}$, release of small molecules and protons ${ }^{16-18}$, stress reporting ${ }^{19-22}$, stress strengthening $22-24$ and soft devices ${ }^{25,26}$. These efforts are largely facilitated by the fact that, unlike other energy sources, mechanical force is directional and regulated by local molecular structure. Even reactions with very similar intrinsic activation barriers can have very different force-coupled reactivities as a result of the structure of the handle through which force is delivered $^{3,8,27-29}$.

Both experimental ${ }^{6,7,24,27,30-38}$ and theoretical ${ }^{29,39-48}$ studies have been conducted to understand force-coupled reactivity and mechanisms, and these investigations provide valuable insights into the design of mechanophore response. One concept for a useful reactivity response is mechanochemical gating, in which one mechanophore (a molecular gate) initially prevents another mechanophore (substrate) from experiencing force delivered along a polymer backbone. When the gate is unlocked mechanically, the substrate feels force and reacts at once in a new type of mechanochemical cascade reaction, reminiscent of the allosteric gating in biological ion transport and signal transmission ${ }^{49-51}$, but driven mechanically rather than by voltage or ligand coupling ${ }^{49-51}$.

Here, we illustrate the gating concept by demonstrating force-gated isomerization of cis-gem-dichlorocyclopropane (cis-gDCC) by cyclobutane in a dual mechanophore, 5,5-dichlorotricyclo(7.2.0.0)undecane (DCTCU). Single-molecule force spectroscopy and ultrasonication experiments reveal that a larger force needs to be applied to a polymer of DCTCU than the polymer of cis-gDCC to isomerize cis-gDCC to dichloroalkene at the same rate. Quantum-chemical calculations confirm that the difference reflects the additional force needed to open the cyclobutane core, that is, that the mechanochemical kinetics of cis-gDCC isomerization is controlled by that of cyclobutane dissociation rather than the intrinsic mechanochemistry of cis-gDCC.

\section{Results}

Computational predictions. The gating concept and our initial system are shown in Fig. 1. Calculations at the uMPW1K/ $6-311+G(d)$ level of DFT with a polarized-continuum model of reaction solvent called CPCM (ref. 52) (used to account for observed solvent effects, Supplementary Table 1) reveal several mechanisms for dissociation of the three scissile bonds of DCTCU (Supplementary Figs 1 and 2) whose relative importance changes with applied force. In the absence of force, the most reactive site of DCTCU is cis-gDCC, whose opening results in a bicyclic intermediate Int1 (Fig. 2a), followed by sequential homolysis of the inner and then of the outer $\mathrm{C}-\mathrm{C}$ bonds of cyclobutane to yield EEE-triene.

Stretching force acting on the $\mathrm{C}$ atoms of the $\mathrm{CH}_{3}$ groups of DCTCU does not affect the apparent activation energy of this path (green line, Fig. 2b), but lowers all barriers of an alternative isomerization mechanism in which the order of dissociation of the 3 scissile bonds is inverted (Fig. 2a). This inversion is a result of the sequential loading of the scissile bonds enforced by the fused-ring architecture of DCTCU (and its homologues): the applied force is coupled to only one scissile bond at a time, which must dissociate before another scissile bond is loaded. Because force strongly accelerates bond dissociation, this sequential loading ensures that bonds break in the same order that they are loaded. For example, when stretching force of $1 \mathrm{nN}$ is applied to DCTCU, the calculated activation free energy for dissociation of the outer $\mathrm{C}-\mathrm{C}$ bond of the cyclobutane moiety (TS1f, blue) is reduced to $32.5 \mathrm{kcal} \mathrm{mol}^{-1}$ compared with $51.7 \mathrm{kcal} \mathrm{mol}^{-1}$ and $41.7 \mathrm{kcal} \mathrm{mol}^{-1}$ for dissociation of the bridgehead bonds of the cyclobutane and cis-gDCC moieties, respectively, which are uncoupled from the applied force (all numbers are for reaction in methylbenzoate solvent). Consequently, the reaction proceeds through TS1f, yielding intermediate Intlf, in which the applied force acting on the second scissile bond of cyclobutane reduces the free-energy barrier of its dissociation to $22.5 \mathrm{kcal} \mathrm{mol}^{-1}$ (TS2f, red). The cis-gDCC moiety in Intlf remains shielded from the force and hence its barrier unchanged at $41.7 \mathrm{kcal} \mathrm{mol}^{-1}$. Only once TS2 $\mathrm{f}$ is traversed is the applied force transmitted to the cis-gDCC moiety, lowering its reaction barrier to $22.6 \mathrm{kcal} \mathrm{mol}^{-1}$ (TS3f, magenta).

In other words, at applied forces where the gating mechanism of Fig. 2a dominates, the rate of $c i s-g D C C$ isomerization is governed by the largest of the activation barrier for dissociation of either the outer (TS1f) or the inner (TS2f) C-C bonds of cyclobutane, rather than by the energy of TS3f. For example at $1 \mathrm{nN}$ in toluene, the rate-determining kinetic barrier for gate dissociation (TS1f) exceeds that of $c i s-g$ DCC isomerization (TS3f) by $9.2 \mathrm{kcal} \mathrm{mol}^{-1}$, corresponding to a $>10^{5}$-fold increase in the

a

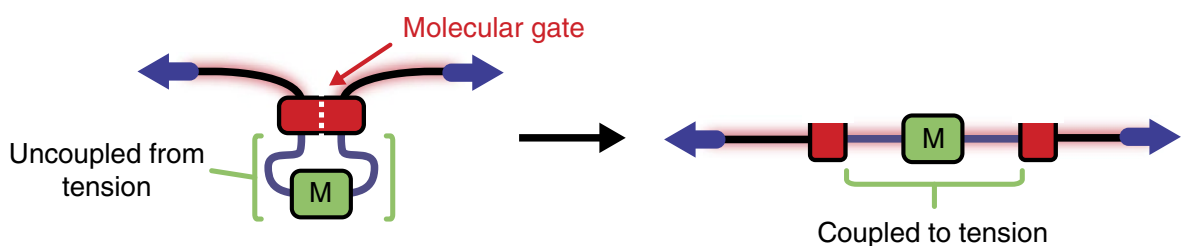

b
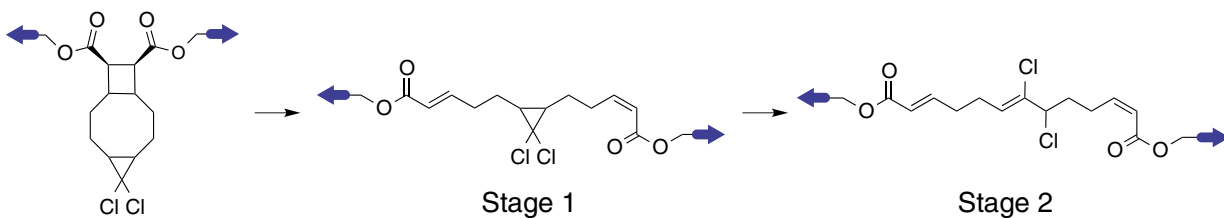

Stage 1

Stage 2

Figure 1 | Schematic representation and molecular design of mechanical reaction gating. (a) A molecular gate (red block) prevents a protected mechanophore substrate (green) from feeling force. Applying sufficiently high force to the gate unlocks it mechanically, allowing the force to be transmitted to the substrate. (b) A cyclobutane mechanophore functions as the gate, and its mechanical cycloreversion unlocks the system so that initially protected cis-gem-dichlorocyclopropane (cis-gDCC) is activated. 
a
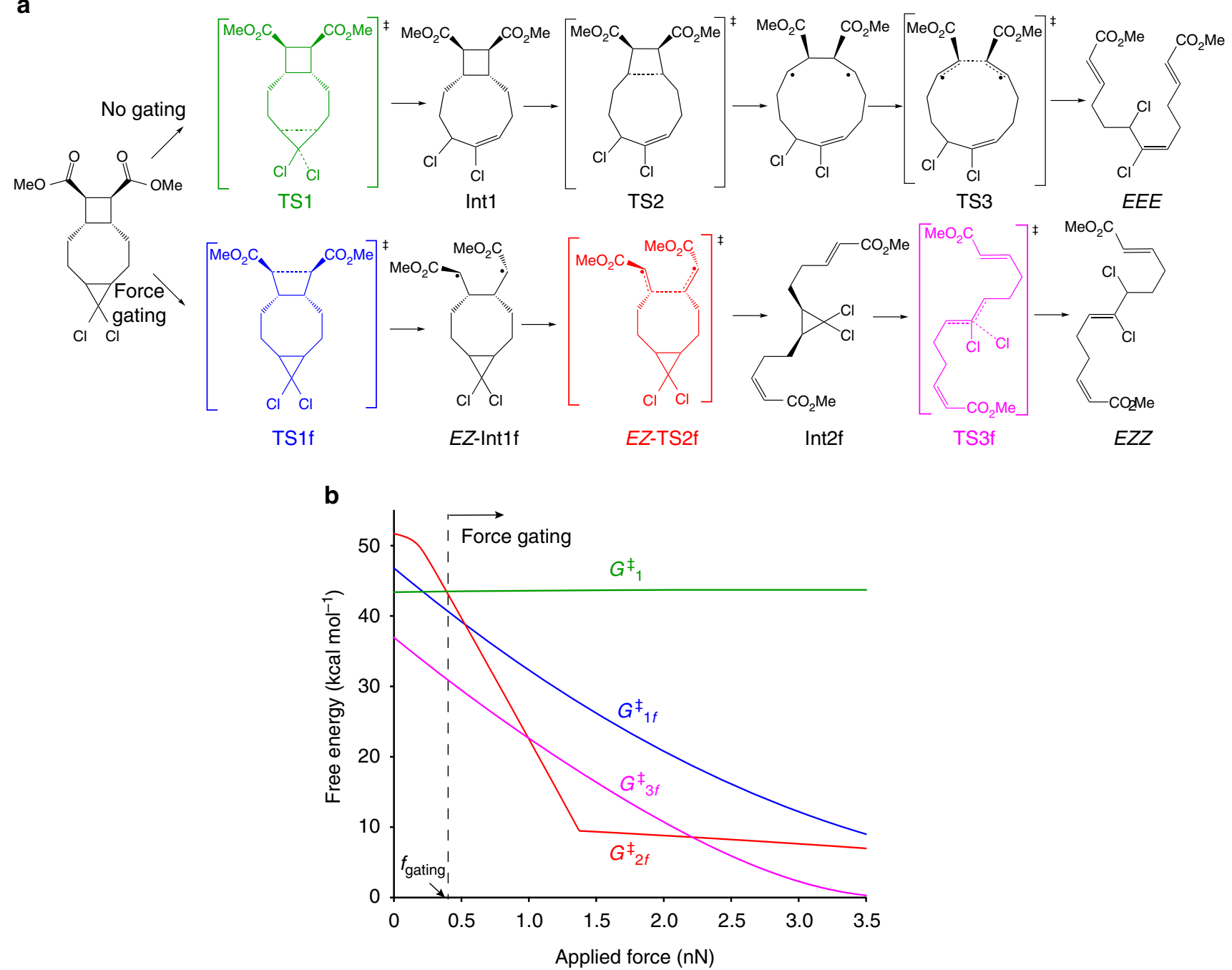

Figure 2 | The computed effects of force on the reaction mechanism and activation barriers. (a) The minimum-energy reaction mechanisms without and with gating are different as are the final products. The relative contributions of the EE and EZ conformers of Int1f and TS2f vary with force (Supplementary Fig. 2): for clarity only one set of isomers is shown. (b) Calculated heights of the free energy barriers (see text for assumptions and details of theoretical methods) in methylbenzoate as a function of the applied force calculated as the differences of the free energies of (i) TS1 and TS1f relative to 5,5-dichlorotricyclo(7.2.0.0) undecane (DCTCU) $\left(G_{1}^{\ddagger}\right.$ and $G_{1 f} \ddagger$, respectively), and (ii) TS3f relative to Int2f $\left(G_{3 f} \frac{\ddagger}{\ddagger}\right) ; G_{2 f} \frac{\ddagger}{\ddagger}$ is the free energy of TS2f relative to (i) DCTCU up to $1.4 \mathrm{nN}$ and (ii) Int1f at $>1.4 \mathrm{nN}$, when Int1f becomes lower in energy than DCTCU. The calculated cross-over between the non-gated and gated reactivity, $f_{\text {gating, }}$ occurs at $\sim 0.45 \mathrm{nN}$ as indicated.

cis-gDCC half-life at room temperature. Equivalently, a polymer of DCTCU would have to be stretched to a force of $1.8 \mathrm{nN}$ to generate the dichloropentene moiety at the same rate as would poly(cis-gDCC) stretched to $1 \mathrm{nN}$.

Single-molecule force spectroscopy. Results of both singlemolecule force spectroscopy (SMFS) and sonication experiments are consistent with gated mechanochemistry of cis-gDCC in DCTCU. The experiments were conducted on polymers containing multiple DCTCUs in the backbone, a strategy that we used in the past to quantify mechanochemistry of gem-dihalocylopropanes $^{7,27,33}$, benzocyclobutenes ${ }^{7}$ and spiropyran ${ }^{53}$. The synthesis (Fig. 3) was similar to that of previously reported bicyclo(4.2.0) octane derivatives $^{34}$. Photochemical $(2+2)$ cycloaddition of maleic anhydride and Z-9,9-dichlorobicyclo(6.1.0)non-4-ene (1) in the presence of benzophenone yielded DCTCU derivative (2), which was esterified with 4-pentenol to diene $\mathbf{3}$ followed by ring closing metathesis ${ }^{54}$ to form macrocycle 4. Entropically-driven ring opening metathesis co-polymerization ${ }^{55-57}$ of 4 with either unsaturated epoxide 5 or cis- $g$ DCC derivative 10 yielded polymer $\mathbf{6}$ and $\mathbf{8}$ for SMFS and sonication experiments, respectively. We incorporated epoxides in polymer $\mathbf{6}$ to increase its adhesion to the tip of atomic force microscope ${ }^{27}$. The identity of all synthetic intermediates and final products were confirmed by ${ }^{1} \mathrm{H}$ and ${ }^{13} \mathrm{C}$ NMR spectroscopy (Supplementary Fig. 3).

The SMFS measurements were conducted at a tip velocity of $300 \mathrm{~nm} \mathrm{~s}^{-1}$ using the previously described procedure ${ }^{7}$. Figure 4 shows a representative measured force-extension curve for polymer $\mathbf{6}$ and for comparison a previously reported ${ }^{27}$ force-extension curve of poly(cis-gDCC) along with the curves extrapolated from the force/activation energy correlations described in Fig. 2b. The average force at the middle of the plateau of the 10 force-extension curves measured was $2.2 \pm 0.15 \mathrm{nN}$, in good agreement with the predicted force of $2.3 \mathrm{nN}$ at the equivalent loading rate. We observed no statistically significant dependence of the measured plateau force on the polymer length, the length of the plateau or the molar fraction of 


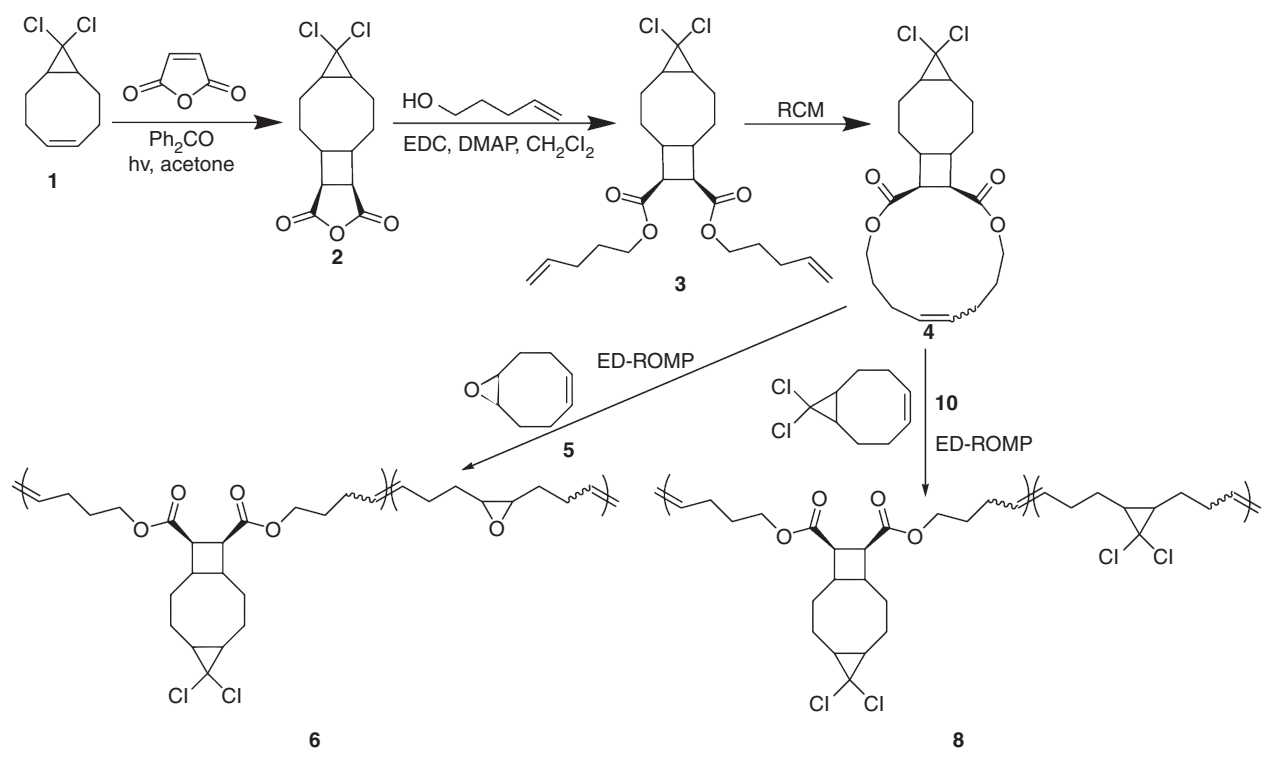

Figure 3 | Chemical structures and synthesis of polymers 6 and 8. Experimental details are provided in the Supplementary Methods; EDC and DMAP are $\mathrm{N}$-(3-dimethylaminopropyl)- $\mathrm{N}^{\prime}$-ethylcarbodiimide hydrochloride and 4-dimethylaminopyridine, respectively. The ring closing metathesis and entropicallydriven ring opening metathesis co-polymerization steps used Grubbs second generation catalysts.

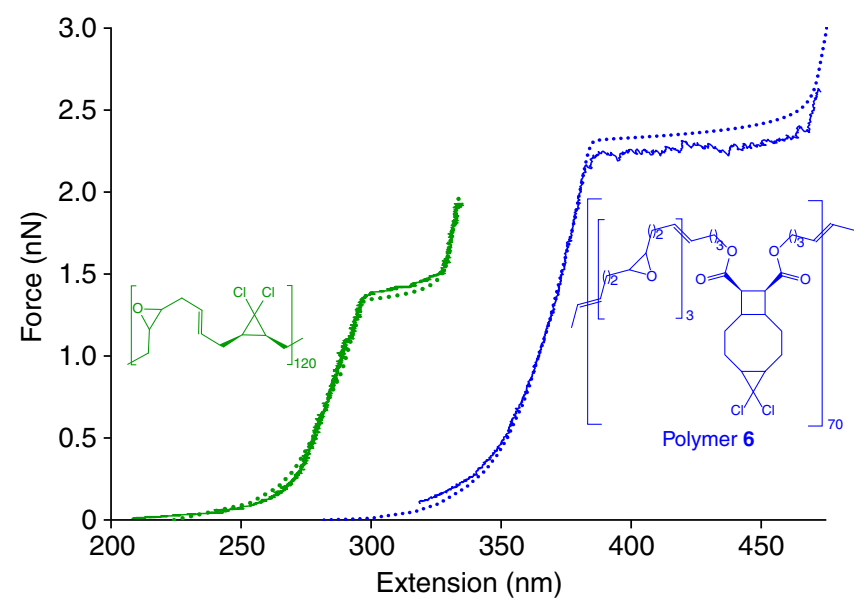

Figure 4 | Measured and computed force-extension curves. The experimental (solid lines) and computational (dotted lines) results of a single macromolecule of polymer $\mathbf{6}$ and of a polymer of cis-gDCC are shown in blue and green, respectively. The molar ratios of epoxide/DCTCU or epoxide/cis-gDCC moieties used to calculate the force-extension curves (red and green structures) were chosen to approximate the ratios measured in bulk polymer samples by ${ }^{1} \mathrm{H}$ NMR. Additional measured force-extension curves are shown in Supplementary Figs 4 and 5 and the computed curves are tabulated in Supplementary Table 3.

the DCTCU moieties measured on bulk samples of polymer 6 by ${ }^{1}$ H NMR spectroscopy (Supplementary Table 2).

The plateau regions of the force-extension curves for $\mathbf{6}$ show greater saw-tooth texture than previously reported curves of other multi-mechanophore polymers ${ }^{7,27,33,53}$, a feature that we attribute to the considerable hidden length (that is, extension generated from ring-opening) of DCTCU. We cannot resolve individual ring-opening events in the force-extension curves, but comparing the length of the plateau with the calculated hidden length of a single DCTCU moiety $(1.2 \mathrm{~nm}$ at $2 \mathrm{nN})$ indicates that in the 10 measured force/extension curves the number of DCTCU units responsible for the observed plateau varied from 11 to 160 , with the median of 46 . The force over which these molecules isomerized varied from $\sim 70$ to $\sim 200 \mathrm{pN}$ but is uncorrelated with the number of reacted molecules, in line with the range reported for other multi-mechanophore polymers, suggesting that even as few as 10 DCTCU molecules is sufficient to approximate the behaviour of an ensemble. The $\sim 575$ isomerization events measured in our SMF experiments all occurred between 2 and $2.4 \mathrm{nN}$, which is consistent with the calculated change in the survival probability of DCTCU under the relevant conditions decreasing from $\sim 0.999$ to $\sim 0.02$ over this range (the values depend slightly on the length of the polymer in each experiment). This contrasts with a recent report ${ }^{58}$ where a total of nine force-accelerated events, one per force-extension curve, attributed to the ring opening of a related cyclobutane derivative occurred at applied forces that were distributed almost uniformly over the $1.7-3.9 \mathrm{nN}$ range. The difference cannot be ascribed to the 15 -fold faster retraction rate and shorter polymers used in ref. 58, but may indicate that the rate-determining barrier of the process studied in ref. 58 is largely independent of the applied force between 1.7 and $3.9 \mathrm{nN}$, similarly to the second barrier of DCTCU isomerization (red curve, Fig. 2b). This speculation, however, remains to be tested computationally and experimentally.

The SMFS behaviour of polymer $\mathbf{6}$ is consistent with the expected gating dynamics. Under comparable conditions the mechanochemical ring-opening in non-gated poly(cis-gDCC) occurs at $1.3 \mathrm{nN}$, or at $>0.8 \mathrm{nN}$ lower force than the plateau of polymer 6. This qualitative observation is further reinforced by the agreement between the measured and calculated forceextension curves for poly(cis-gDCC) and polymer 6 (Fig. 4). Both theory and experiment, therefore, support cyclobutane-gating of mechanochemistry of cis-gDCC in polymer $\mathbf{6}$.

Sonication experiments. Our QM calculations suggest that mechanical gating should be retained even at forces in the $>3 \mathrm{nN}$ range characteristic of polymer mechanochemistry during pulsed ultrasonication, which was indeed confirmed with the products characterized by ${ }^{1} \mathrm{H}$-NMR spectroscopy. As shown in Fig. $2 \mathrm{~b}$, in DCTCU, mechanochemical isomerization of cis-gDCC can only 
occur after complete dissociation of the cyclobutane, and the activation energy for cis-gDCC isomerization is much lower than that for the cyclobutane gating reaction at the same applied force. This means that the molar fractions of cis-gDCC and cyclobutane moieties that ring open during sonication should be identical. ${ }^{1} \mathrm{H}$ NMR spectra of THF solutions of polymer $\mathbf{6}$ subjected to pulsed ultrasonication (at power density of $11.9 \mathrm{~W} \mathrm{~cm}^{-2}$ ) showed the signals of $E$ and $Z$ isomers of $\alpha, \beta$-unsaturated ester $(\delta \sim 5.8,6.2$ and 6.9 p.p.m. $)$ and of the $Z$-2,3-dichloroalkene $(\delta \sim 4.5$ and 5.8 p.p.m.), expected from ring opening of cyclobutane $e^{34}$ and cis-gDCC (ref. 59), respectively (Fig. 5a,c; DCTCU has no peaks at these chemical shifts). Integration of the peaks (Fig. 5d, Supplementary Fig. 6) shows that the fraction of ring opening products for cyclobutane and cis-gDCC was identical, consistent with the expected sequential activation.

To further validate the mechanical gating mechanism, random copolymers of DCTCU with cis-gDCC (polymer 8, Fig. 5b) were synthesized and sonicated (Supplementary Fig. 7). As shown in Fig. 5e, in contrast to the pure DCTCU polymer $\mathbf{6}$, sonication of 8 produced $\sim 3.5$ cis-gDCC ring opening events per cyclobutane scission. Since as established above this ratio is $1: 1$ for mechanochemical isomerization of DCTCU, the 2.5-fold excess of cis-gDCC ring opening reflects the higher reactivity of ungated cis-gDCC than the gated one, because the force-coupled reactivity of cis-gDCC during sonication is greater than cyclobutane, as predicted by our computations. The cyclobutane gate therefore determines the mechanochemical reactivity of cis- $g \mathrm{DCC}$ across the force range accessed in both SMFS and sonication, in both situations increasing the applied force needed to lower the halflife of cis-gDCC to the relevant timescale of each experiment.

\section{Discussion}

The gating concept applied here is deceptive in its apparent simplicity, and we illustrate some important subtleties on the example of DCTCU. First, gating requires that dissociation of the gate traverse a higher barrier than that of the substrate $(g D C C$ in our case) at the same force. Otherwise, the rate at which the substrate reacts in the loaded mechanophore will be controlled by the intrinsic mechanochemical kinetics of the substrate and will be identical to that of non-gated analogue. DCTCU meets this condition over all forces, as evidenced by the transition state of $g$ DCC isomerization (magenta curve in Fig. 2b) being more stable than at least one of the transition states of cyclobutane dissociation (blue and red curves). Because of the high strain-free activation free energy of dissociation of cyclobutane, it is likely to be suitable for gating a diverse range of mechanochemical reactions up to $\mathrm{nN}$-range forces.

Second, gating requires that the rate-determining activation barrier of dissociation of the gating moiety be less than the

a

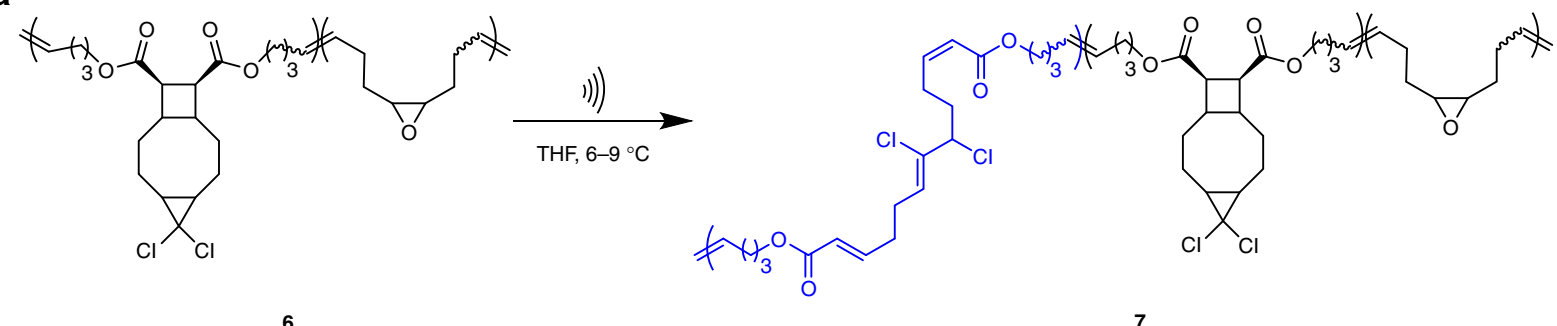

b

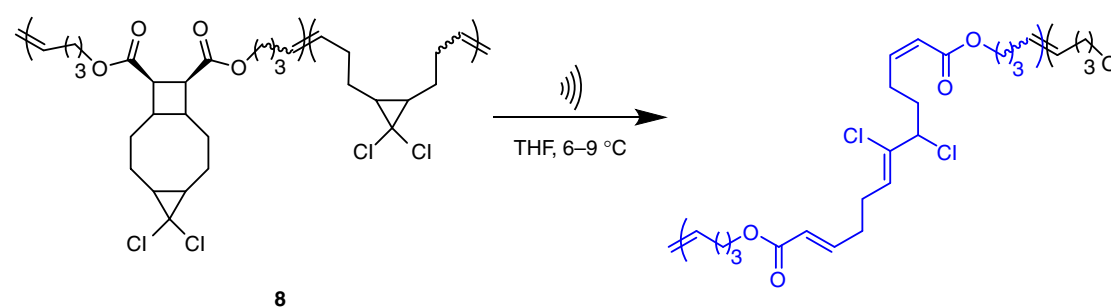

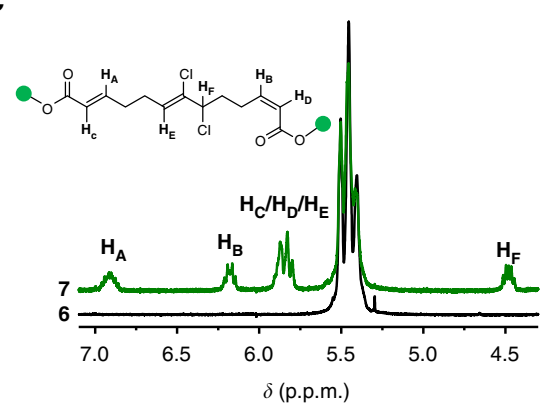

d

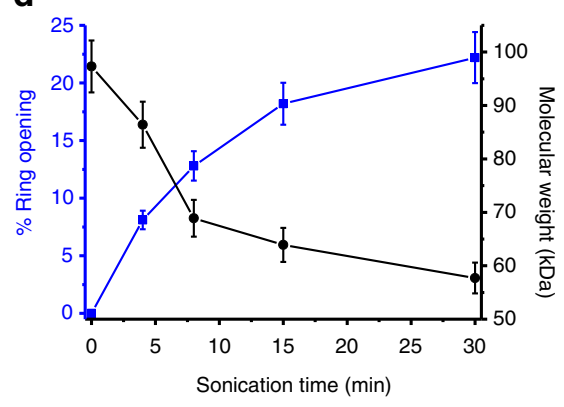

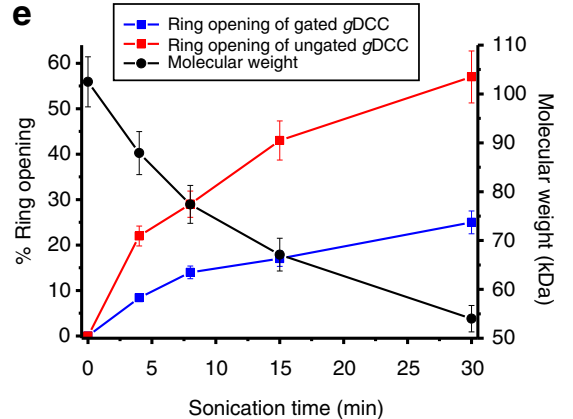

Figure 5 | Sonication of copolymers of DCTCU with epoxide (6) and cis-gDCC (8). (a,b) Primary chemical reactions resulting from sonication of $\mathbf{6}$ (a) and $\mathbf{8}$ (b). Sonication of $\mathbf{8}$ leads to ring opening of $g$ DCC gated within the initial DCTCU (blue fragment in 9) and ungated along the polymer backbone (red fragment). (c) Partial ${ }^{1} \mathrm{H}$ NMR spectrum of polymer $\mathbf{6}$ before (black) and after (green) sonication. (d,e) Molar fractions of isomerized DCTCU moieties (blue, polymers $\mathbf{6}$ and $\mathbf{8}$ ) and backbone gDCC moieties (red, polymer $\mathbf{8}$ only) as a function of sonication time of polymer $\mathbf{6}$ (d) and $\mathbf{8}$ (e). As is typical for every other polymer reported, non-selective backbone fragmentation competes with mechanophore-centered chemistry during sonication of polymers 6 and 8, decreasing their average molecular weights (black axes and Supplementary Figs 8 and 9). Error bars are standard deviations. 


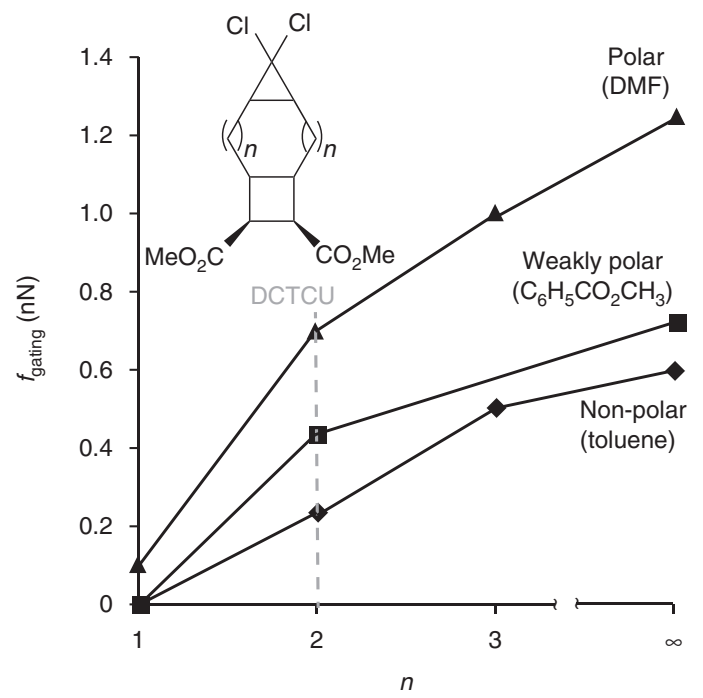

Figure 6 | Gating as a function of intervening ring size. The calculated $f_{\text {gating }}$ (see text for assumptions and computational details) depends on solvent polarity and the size of the separating ring, $n$. Data for $n=\infty$ corresponds to a central ring large enough to impose no compressive strain in corresponding TS1 (green structure, Fig. 1a) and was extrapolated using force-dependent activation free energy of ring opening of cis-1,1-dichloro2,3-dimethylcyclopropane.

activation barrier of the unloaded substrate. Otherwise, the unloaded substrate will react preferentially, as happens in DCTCU at applied force $<0.4 \mathrm{nN}$ (in methylbenzoate). In DCTCU this minimum gating force, $f_{\text {gating, }}$, is determined by the difference of the kinetic barriers for dissociation of $g \mathrm{DCC}$ and of cyclobutane (that is, TS1 versus the highest of TS1f or TS2f). This difference is sensitive to both the reaction solvent and the size of the ring separating the cyclobutane and $g \mathrm{DCC}$ moieties, because these variables affect the energy of TS1 but not of TS1f or TS2f. Polar solvents stabilize TS1 (for example, its energy is $39.8 \mathrm{kcal} \mathrm{mol}^{-1}$ and $47.9 \mathrm{kcal} \mathrm{mol}^{-1}$ in DMF and toluene, respectively), whereas smaller rings destabilize it, by imposing a larger compressive strain on it (for example, shrinking the central ring of DCTCU by 2 atoms to yield 4,4-dichlorotricyclo (5.2.0.0)nonane, $n=1$, Fig. 6 increases TS1 by $12.5 \mathrm{kcal} \mathrm{mol}^{-1}$ in toluene). By varying these two parameters, $f_{\text {gating }}$ can be changed systematically from $0 \mathrm{nN}$ up to $\sim 1.3 \mathrm{nN}$, as may be required for a particular application of the gated mechanophore. For example, in DCTCU $(n=2$, Fig. 6), changing the reaction solvent from toluene to DMF increases $f_{\text {gating }}$ from 0.2 to $0.7 \mathrm{nN}$. A very large ring separating the $g \mathrm{DCC}$ and cyclobutane moieties $(n>3)$ would shift this range up to $0.6-1.3 \mathrm{nN}$.

Lastly, force also affects the geometry of the $3 \mathrm{C}=\mathrm{C}$ bonds generated by ring opening of DCTCU. The geometry of the central $\mathrm{C}=\mathrm{C}$ bond is determined by whether the $g \mathrm{DCC}$ or cyclobutane ring opened first: at force below $f_{\text {gating, }}$, the reaction proceeds through TS1, yielding the $E$ isomer, whereas at higher forces $g \mathrm{DCC}$ isomerizes through TS3f, producing the $\mathrm{Z}$ configuration. The geometries of the $2 \mathrm{C}=\mathrm{C}$ bonds generated by dissociation of cyclobutane are determined by the relative energies of the conformers of TS2f, all of which are accessible by low-barrier rotation around the two exocyclic bonds of Intlf: at force $<0.15 \mathrm{nN}$, the $E E$ conformer is lowest-energy, while the $E Z$ conformer dominates at higher forces (Supplementary Fig. 2). Consequently, depending on applied force, DCTCU yields one of the three isomeric trienes as the major product: $E E E$ at $0-0.15 \mathrm{nN}$; $E E Z$ at $0.15-0.4 \mathrm{nN}$ (in methylbenzoate) and $E Z Z$ at higher force.
While the generation of the EZZ product at $2+\mathrm{nN}$ is consistent with our experimental results discussed above, as well as the previously reported data for mechanochemical isomerization of bicyclo(4.2.0)octane derivatives ${ }^{34}$, the very high activation barriers of isomerization at forces $<0.4 \mathrm{nN}$ have so far prevented us from confirming the calculated mechanism at low forces.

In conclusion, we designed a mechanical gating system, in which one mechanophore functions as a gate to modulate the reactivity of another mechanophore, resulting in a mechanochemical cascade reaction. Both SMFS and sonication results are consistent with the sequential activation. The concept of mechanical gating can be applied to situations where controlled reactivity is desired, including catalysis, small molecule release and stress sensing. In addition, we quantified the mechanochemical kinetics of the cycloreversion of the increasingly popular cyclobutane mechanophore motif ${ }^{60-62}$, and therefore expanded the small database of quantitative measures of mechanochemical reactivity.

\section{Methods}

SMFS. On a piece of $1 \times 1 \mathrm{~cm}^{2}$ silicon substrate surface was added $20 \mu \mathrm{l}$ $0.5 \mathrm{mg} \mathrm{ml}^{-1}$ THF solution of the polymer, and the surface was left to dry, allowing the polymer to be absorbed on the surface. The substrate was mounted on the top of a piezo scanner, and the AFM tip was brought into contact with the surface and then retracted from it. The tip velocity was set at $300 \mathrm{~nm} \mathrm{~s}^{-1}$ and the approaching/ retracting cycles were recorded. The experiments were conducted in both methyl benzoate and toluene, in different days with different cantilever tips.

QM calculation. Free energies in Fig. 2 were calculated at the uMPW1K/6$311+\mathrm{G}(\mathrm{d})$ level of the DFT in CPCM model of the reaction solvent using complete conformational ensembles in pseudo-harmonic approximation ${ }^{35,63}$. The model chemistry was chosen because it reproduced the experimental free energies of activation of isomerization of cis-2,3-dimethyl-1,1-dichlorocyclopropane in DMF and diphenyl ether, and of the electronic activation energies of dissociation of $c i s$ cyclobutane-1,2-dicarboxylic acid and isomerization of cis-2,3-dimethyl-1,1dichlorocyclopropane in vacuum calculated at the uCCSD/6-31G(d)//uCCSD/ jun-cc-pVTZ and uCCSD/6-311 + G(d)//uCCSD $(\mathrm{T}) /$ jun-cc-pVTZ levels of theory, respectively (Supplementary Tables 1 and 4). Force-extension curves were calculated using the previously reported approach ${ }^{37}$ by combining the force-dependent activation free energies and force-extension correlations of a single repeat unit of polymer 6 (blue curves, Fig. 4) or 2 repeat units of poly(cis-gDCC) (green curves) in the reactant and the product states, calculated using partial conformational ensembles of 3 lowest-energy conformers for each state. The loading rate used to calculate the force-extension curves was $2-6 \mathrm{~nm} \mathrm{~s}^{-1}$ (depending on the length of the polymer in the corresponding experiment), to reproduce the time that the plateau was traversed in SMFS experiments. The calculated force-extension curves were then scaled to reproduce the shape of the experimental curves between 0.3 and $1.6 \mathrm{nN}$ (for polymer 6) or between 0.2 and $1 \mathrm{nN}$ (for poly(cis-gDCC)).

Data availability. The data sets generated during and/or analysed during the current study are available from the corresponding authors on reasonable request.

\section{References}

1. Caruso, M. M. et al. Mechanically-induced chemical changes in polymeric materials. Chem. Rev. 109, 5755-5798 (2009).

2. Ribas-Arino, J. \& Marx, D. Covalent mechanochemistry: theoretical concepts and computational tools with applications to molecular nanomechanics. Chem. Rev. 112, 5412-5487 (2012).

3. Brown, C. L. \& Craig, S. L. Molecular engineering of mechanophore activity for stress-responsive polymeric materials. Chem. Sci. 6, 2158-2165 (2015).

4. Li, J., Nagamani, C. \& Moore, J. S. Polymer mechanochemistry: from destructive to productive. Accts. Chem. Res. 48, 2181-2190 (2015).

5. Huang, Z. \& Boulatov, R. Chemomechanics: chemical kinetics for multiscale phenomena. Chem. Soc. Rev. 40, 2359-2384 (2011).

6. Hickenboth, C. R. et al. Biasing reaction pathways with mechanical force. Nature 446, 423-427 (2007).

7. Wang, J. et al. Inducing and quantifying forbidden reactivity with singlemolecule polymer mechanochemistry. Nat. Chem. 7, 323-327 (2015).

8. Wang, J., Kouznetsova, T. B., Niu, Z., Rheingold, A. L. \& Craig, S. L. Accelerating a mechanically driven anti-Woodward-Hoffmann ring opening with a polymer lever arm effect. J. Org. Chem. 80, 11895-11898 (2015).

9. Wollenhaupt, M., Krupička, M. \& Marx, D. Should the Woodward-Hoffmann rules be applied to mechanochemical reactions? ChemPhysChem. 16, 1593-1597 (2015). 
10. Lenhardt, J. M. et al. Trapping a diradical transition state by mechanochemical polymer extension. Science 329, 1057-1060 (2010).

11. Klukovich, H. M. et al. Tension trapping of carbonyl ylides facilitated by a change in polymer backbone. J. Am. Chem. Soc. 134, 9577-9580 (2012).

12. Piermattei, A., Karthikeyan, S. \& Sijbesma, R. P. Activating catalysts with mechanical force. Nat. Chem. 1, 133-137 (2009).

13. Jakobs, R. T. M. \& Sijbesma, R. P. Mechanical activation of a latent olefin metathesis catalyst and persistence of its active species in romp. Organometallics 31, 2476-2481 (2012).

14. Kean, Z. S. et al. Photomechanical actuation of ligand geometry in enantioselective catalysis. Angew. Chem. Intl. Ed. 53, 1-5 (2014).

15. Clough, J. M., Balan, A. \& Sijbesma, R. P. Mechanochemical reactions reporting and repairing bond scission in polymers. Top. Curr. Chem. 369, 209-238 (2015).

16. Diesendruck, C. E. et al. Proton-coupled mechanochemical transduction: A mechanogenerated acid. J. Am. Chem. Soc. 134, 12446-12449 (2012).

17. Larsen, M. B. \& Boydston, A. J. 'Flex-activated' mechanophores: using polymer mechanochemistry to direct bond bending activation. J. Am. Chem. Soc. 135, 8189-8192 (2013).

18. Gossweiler, G. R. et al. Mechanochemical activation of covalent bonds in polymers with full and repeatable macroscopic shape recovery. ACS Macro Lett. 3, 216-219 (2014).

19. Davis, D. A. et al. Force-induced activation of covalent bonds in mechanoresponsive polymeric materials. Nature 459, 68-72 (2009).

20. Chen, Y. et al. Mechanically induced chemiluminescence from polymers incorporating a 1,2-dioxetane unit in the main chain. Nat. Chem. 4, 559-562 (2012).

21. Ducrot, E., Chen, Y., Bulters, M., Sijbesma, R. P. \& Creton, C. Toughening elastomers with sacrificial bonds and watching them break. Science 344, 186-189 (2014).

22. Verstraeten, F., Gostl, R. \& Sijbesma, R. P. Stress-induced colouration and crosslinking of polymeric materials by mechanochemical formation of triphenylimidazolyl radicals. Chem. Commun. 52, 8608-8611 (2016).

23. Wang, J., Piskun, I. \& Craig, S. L. Mechanochemical strengthening of a multi-mechanophore benzocyclobutene polymer. ACS Macro Lett. 4, 834-837 (2015).

24. Zhang, H. et al. Mechanochromism and mechanical-force-triggered crosslinking from a single reactive moiety incorporated into polymer chains. Angew. Chem. Int. Ed. 55, 3040-3044 (2016).

25. Wang, Q., Gossweiler, G. R., Craig, S. L. \& Zhao, X. Cephalopod-inspired design of electro-mechano-chemically responsive elastomers for on-demand fluorescent patterning. Nat. Commun. 5, 4899 (2014).

26. Gossweiler, G. R. et al. Mechanochemically active soft robots. ACS Appl. Mater. Interfaces 7, 22431-22435 (2015).

27. Klukovich, H. M., Kouznetsova, T. B., Kean, Z. S., Lenhardt, J. M. \& Craig, S. L. A backbone lever-arm effect enhances polymer mechanochemistry. Nat. Chem. 5, 110-114 (2013).

28. Wang, J. et al. A remote stereochemical lever arm effect in polymer mechanochemistry. J. Am. Chem. Soc. 136, 15162-15165 (2014).

29. Tian, Y. \& Boulatov, R. Quantum-chemical validation of the local assumption of chemomechanics for a unimolecular reaction. ChemPhysChem. 13, 2277-2281 (2012)

30. Kersey, F. R., Yount, W. C. \& Craig, S. L. Single-molecule force spectroscopy of bimolecular reactions: system homology in the mechanical activation of ligand substitution reactions. J. Am. Chem. Soc. 128, 3886-3887 (2006).

31. Wiita, A. P., Ainavarapu, R. K., Huang, H. H. \& Fernandez, J. M. Force-dependent chemical kinetics of disulfide bond reduction observed with single-molecule techniques. Proc. Natl. Acad. Sci. (USA) 103, 7222-7227 (2006).

32. Schmidt, S. W., Beyer, M. K. \& Clausen-Schaumann, H. Dynamic strength of the silicon - carbon bond observed over three decades of force-loading rates. J. Am. Chem. Soc. 130, 3664-3668 (2008).

33. Wu, D., Lenhardt, J. M., Black, A. L., Akhremitchev, B. B. \& Craig, S. L. Molecular stress relief through a force-induced irreversible extension in polymer contour length. J. Am. Chem. Soc. 132, 15936-15938 (2010).

34. Kean, Z. S., Niu, Z., Hewage, G. B., Rheingold, A. L. \& Craig, S. L. Stress-responsive polymers containing cyclobutane core mechanophores: reactivity and mechanistic insights. J. Am. Chem. Soc. 135, 13598-13604 (2013).

35. Tian, Y., Kucharski, T. J., Yang, Q.-Z. \& Boulatov, R. Model studies of force-dependent kinetics of multi-barrier reactions. Nat. Commun 4, 2538 (2013).

36. Akbulatov, S., Tian, Y., Kapustin, E. \& Boulatov, R. Model studies of the kinetics of ester hydrolysis under stretching force. Angew. Chem. Int. Ed. 52, 6992-6995 (2013).

37. Akbulatov, S., Tian, Y. \& Boulatov, R. Force-reactivity property of a single monomer is sufficient to predict the micromechanical behavior of its polymer. J. Am. Chem. Soc. 134, 7620-7623 (2012).
38. Li, Y. C. \& Sheiko, S. S. Molecular mechanochemistry: engineering and implications of inherently strained architectures. Top. Curr. Chem. 369, 1-36 (2015).

39. Ong, M. T., Leiding, J., Tao, H., Virshup, A. M. \& Martínez, T. J. First principles dynamics and minimum energy pathways for mechanochemical ring opening of cyclobutene. J. Am. Chem. Soc. 131, 6377-6379 (2009).

40. Dopieralski, P. et al. The Janus-faced role of external forces in mechanochemical disulfide bond cleavage. Nat. Chem. 5, 685-691 (2013).

41. Dopieralski, P., Ribas-Arino, J., Anjukandi, P., Krupicka, M. \& Marx, D. Force-induced reversal of beta-eliminations: stressed disulfide bonds in alkaline solution. Angew. Chem. Intl. Ed. 55, 1304-1308 (2016).

42. Hermes, M. \& Boulatov, R. The entropic and enthalpic contributions to force-dependent dissociation kinetics of the pyrophosphate bond. J. Am. Chem. Soc. 133, 20044-20047 (2011).

43. Tian, Y. \& Boulatov, R. Comparison of the predictive performance of the bell-evans, taylor-expansion and statistical-mechanics models of mechanochemistry. Chem. Commun. 49, 4187-4189 (2013).

44. Kucharski, T. J. \& Boulatov, R. The physical chemistry of mechanoresponsive polymers. J. Mater. Chem. 21, 8237-8255 (2011).

45. Kochhar, G. S., Bailey, A. \& Mosey, N. J. Competition between orbitals and stress in mechanochemistry. Angew. Chem. Int. Ed. 49, 7452-7455 (2010).

46. Suzuki, Y. \& Dudko, O. K. Single-molecule rupture dynamics on multidimensional landscapes. Phys. Rev. Lett. 104, 048101 (2010).

47. Huang, Z. \& Boulatov, R. Chemomechanics with molecular force probes. Pure Appl. Chem. 82, 931-951 (2010).

48. Konda, S. S. M., Brantley, J. N., Bielawski, C. W. \& Makarov, D. E. Chemical reactions modulated by mechanical stress: extended bell theory. J. Chem. Phys 135, 164103 (2011)

49. May, L. T., Leach, K., Sexton, P. M. \& Christopoulos, A. Allosteric modulation of G protein-coupled receptors. Ann. Rev. Pharm. Toxicology 47, 1-51 (2007).

50. Osteen, J. D. et al. Allosteric gating mechanism underlies the flexible gating of KCNQ1 potassium channels. Proc. Natl Acad. Sci. (USA) 109, 7103-7108 (2012).

51. Koh, J. \& Blobel, G. Allosteric regulation in gating the central channel of the nuclear pore complex. Cell 161, 1361-1373 (2015).

52. Tomasi, J., Mennucci, B. \& Cammi, R. Quantum mechanical continuum solvation models. Chem. Rev. 105, 2999-3094 (2005).

53. Gossweiler, G. R., Kouznetsova, T. B. \& Craig, S. L. Force-rate characterization of two spiropyran-based molecular force probes. J. Am. Chem. Soc. 137, 6148-6151 (2015).

54. Lee, C. W. \& Grubbs, R. H. Stereoselectivity of macrocyclic ring-closing olefin metathesis. Org. Lett. 2, 2145-2147 (2000).

55. Hodge, P. \& Kamau, S. D. Entropically driven ring-opening-metathesis polymerization of macrocyclic olefins with 21-84 ring atoms. Angew. Chem. Int. Ed. 42, 2412-2414 (2003).

56. Tastard, C. Y., Hodge, P., Ben-Haida, A. \& Dobinson, M. Entropically driven ring-opening metathesis polymerization (ed-romp) of macrocyclic olefincontaining oligoamides. React. Funct. Polymers 66, 93-107 (2006).

57. Kamau, S. D., Hodge, P., Hall, A. J., Dad, S. \& Ben-Haida, A. Cyclodepolymerization of olefin-containing polymers to give macrocyclic oligomers by metathesis and the entropically-driven romp of the olefin-containing macrocyclic esters. Polymer 48, 6808-6822 (2007).

58. Pill, M. F. et al. Mechanochemical cycloreversion of cyclobutane observed the single molecule level. Chem. Eur. J 22, 12034-12039 (2016).

59. Lenhardt, J. M., Black, A. L. \& Craig, S. L. Gem-dichlorocyclopropanes as abundant and efficient mechanophores in polybutadiene copolymers under mechanical stress. J. Am. Chem. Soc. 131, 10818-10819 (2009).

60. Klukovich, H. M., Kean, Z. S., Iacono, S. T. \& Craig, S. L. Mechanically induced scission and subsequent thermal remending of perfluorocyclobutane polymers. J. Am. Chem. Soc. 133, 17882-17888 (2011).

61. Kryger, M. J., Munaretto, A. M. \& Moore, J. S. Structure-mechanochemical activity relationships for cyclobutane mechanophores. J. Am. Chem. Soc. 133, 18992-18998 (2011).

62. Kryger, M. J. et al. Masked cyanoacrylates unveiled by mechanical force. J. Am. Chem. Soc. 132, 4558-4559 (2010).

63. Cramer, C. J. Essentials of Computational Chemistry: Theories and Models (Wiley, 2013).

\section{Acknowledgements}

This material is based on work supported by the National Science Foundation under grant CHE-1508566 (to S.L.C.), by EPSRC (EP/L000075/1) and the Royal Society (to R.B.) and by XSEDE (to S.L.C. and R.B.). The authors acknowledge the facilities supported by NSF Research Triangle MRSEC (DMR-1121107). The authors thank G. Gossweiler for assistance in making figures, and C. Brown for measuring the kinetics of $g$ DCC isomerization in different solvents 


\section{Author contributions}

J.W. and R.B. conceived the idea. J.W. and S.L.C. and designed the experiments. J.W. performed the synthesis and sonication experiments. T.B.K. performed the SMFS experiments and collected the data. R.B. performed the QM calculation. J.W., R.B. and S.L.C. analysed the data and wrote the manuscript.

\section{Additional information}

Supplementary Information accompanies this paper at http://www.nature.com/ naturecommunications

Competing financial interests: The authors declare no competing financial interests.

Reprints and permission information is available online at http://npg.nature.com/ reprintsandpermissions/
How to cite this article: Wang, J. et al. Mechanical gating of a mechanochemical reaction cascade. Nat. Commun. 7, 13433 doi: 10.1038/ncomms13433 (2016).

Publisher's note: Springer Nature remains neutral with regard to jurisdictional claims in published maps and institutional affiliations.

(c) (i) This work is licensed under a Creative Commons Attribution 4.0 International License. The images or other third party material in this article are included in the article's Creative Commons license, unless indicated otherwise in the credit line; if the material is not included under the Creative Commons license, users will need to obtain permission from the license holder to reproduce the material. To view a copy of this license, visit http://creativecommons.org/licenses/by/4.0/

C) The Author(s) 2016 